\title{
AFTERWORD: COVID-19 AND HEALTH INEQUITIES
}

As this text approaches publication, we find ourselves in a strange, new world where the very fabric of society has been turned on end by a virus that is $120 \mathrm{~nm}$ in size, but seismic in its impact. Strangers and loved ones have become potential vectors, groceries are potential fomites, and we are physically shielding ourselves from the outside world to a degree no one alive has ever seen.

At the same time COVID-19 has changed the world, it has also further entrenched many of the issues discussed throughout this book. The headlines that are grabbing attention worldwide are not new to anyone in the field of health equity, and many of us knew the day would come where the dire inequities discussed throughout this text revealed themselves in newly disturbing ways as a result of the pandemic. As we go to press, we are only beginning to see the magnitude of disparities in outcomes, with initial state reports showing African American populations facing double, sometimes even triple, the risk of dying in comparison to their White counterparts. Major media outlets are covering this disparity as if it were some new horror inflicted upon the United States, but it is just the latest manifestation of health inequities.

While the full ways in which COVID-19 has exacerbated inequities will not be known for many years - if ever-there are already marked issues that have emerged. Many states now acknowledge that minority populations are much more likely to die from COVID-19. One of the current prevailing theories maps back to everything discussed in these pages: The social determinants of health that lead to inequities in outcomes ranging from cancer to heart disease bear out in the same way with this new pandemic. Lack of transportation, higher rates of uninsurance, poverty, lack of a primary care provider-all of these factors place minority communities at particular risk for negative outcomes. In addition, the cumulative impact of these inequities-for instance, diabetes and hypertension-are in and of themselves risk factors. So the virus reveals what many of us have seen for years: individual health inequities compounding to create accumulative, synergistic inequities of even higher magnitude. For instance, we know that maternal mortality rates are up to five times higher for African American women, with factors ranging from social determinants of health to disparities in underlying health status contributing to this massive level of risk. The same applies to COVID-19: A population already facing an increased likelihood of nearly every identified risk factor for negative COVID-19 outcome (e.g., asthma, heart conditions, obesity, diabetes, chronic kidney disease, liver disease) will inevitably face a higher mortality risk than those not facing the same risk factors and preexisting conditions.

At the same time, members of minority groups not directly infected with COVID-19 are still unequally impacted by it. Minority groups are disproportionately represented among numerous categories of essential workers, including grocery store and supermarket clerks, convenience store 
workers, and food processing plant workers. These high-touch and close-proximity jobs not only placed workers at higher risk for being exposed to COVID-19 prior to shutdown and shelter-inplace ordinances, the subsequent designation of their jobs (understandably) as essential requires them to continue to place themselves at risk for the "benefit of society." Thus, the nature of employment is inherently increasing risk for minority Americans.

The impact is deeper, however, as even the designated actions to prevent spread further reveal the depth of privilege in the United States. Minority populations are less represented in managerial and professional occupations that lend themselves to telecommuting and are less likely to have access to the home broadband necessary to telecommute even if it is an option. This impacts not only work-from-home capabilities but also access to telehealth and home-based education for children. While figures have not been published as of this text going to press, because of these factors, it is highly likely that unemployment claims will include a disproportionate representation of minority residents, and the financial fallout could have years-if not decades-of impact. This will only worsen the already dire inequities that exist and will likely result in even more pronounced health inequities moving forward unless decisive action is taken.

Issues with discrimination are also becoming more prominent. Even early in the outbreak, Asian Americans reported increased numbers of verbal and even physical attacks due to their heritage. The effects of racism in the age of COVID-19 can also be more sinister and emerge in ways that further heighten risk for minority groups. For example, the control strategy of wearing a mask in public further reveals privilege as African American men in particular recount multiple instances of profiling and even harassment for following federal guidelines of wearing masks in public. Many news stories have spotlighted minority men who are electing not to wear masks in light of this, which further increases their risk. Thus, experiences of discrimination are not only becoming more frequent, the effects of racism are also directly increasing risk of infection.

Finally, in states that have begun reopening strategies, the industries initially released often include a high relative proportion of minority workers-for example, barbershops, hair salons, and nail salons. Some officials have even described opening these industries as test cases, despite the known disproportionate impact these actions will have on minority groups. It is unclear what effect this will have on continuing the inequities in COVID-19 outcomes that are already being revealed, but it is difficult to see how things will not worsen.

We are in strange times, and these times are highlighting and exacerbating the factors discussed throughout this text. We can only hope that as a country we seek to find solutions that will lessen, rather than heighten, the impact of health inequities. 